\title{
Monotone iterates for solving systems of semilinear elliptic equations and applications \\ I. Boglaev ${ }^{1}$
}

(Received 20 July 2007; revised 29 April 2008)

\begin{abstract}
Consider monotone finite difference iterative algorithms for solving coupled systems of semilinear elliptic equations. A monotone domain decomposition algorithm based on a modification of Schwarz alternating method and on decomposition of a computational domain into nonoverlapping subdomains is constructed. Advantages of the algorithm are that the algorithm solves only linear discrete systems at each iterative step, converges monotonically to the exact solution of the nonlinear discrete problem, and is potentially parallelisable. The monotone domain decomposition algorithm is applied to a gas-liquid interaction model. Numerical experiments confirm theoretical results.
\end{abstract}

\section{Contents}

1 Introduction

See http://anziamj.austms.org.au/ojs/index.php/ANZIAMJ/article/view/311 for this article, (C) Austral. Mathematical Soc. 2008. Published June 9, 2008. ISSN 14468735 
2 A nonlinear difference scheme

C593

3 Monotone domain decomposition algorithm

C595

3.1 Statement of domain decomposition algorithm . . . . . . C595

3.2 Monotone convergence of algorithm (4)-(6) . . . . . . . C598

4 A gas-liquid interaction model

C602

4.1 Numerical example . . . . . . . . . . . . . . . . . . C604

References

C606

\section{Introduction}

The elliptic system under consideration is in the form

$$
\begin{aligned}
& -\nabla \cdot\left(a_{i} \nabla u_{i}\right)+f_{i}\left(x, u_{1}, u_{2}\right)=0, \quad x \in \omega, \quad i=1,2, \\
& u_{i}(x)=g_{i}(x), \quad i=1,2, \quad x \in \partial \omega, \\
& x \in \mathbb{R}^{k}, \quad \bar{\omega}=\prod_{\alpha=1}^{k} \bar{\omega}^{x_{\alpha}}, \quad \bar{\omega}^{x_{\alpha}}=\left\{0 \leq x_{\alpha} \leq r_{\alpha}\right\},
\end{aligned}
$$

where $\bar{\omega}=\omega \cup \partial \omega, \partial \omega$ is the boundary and $\nabla$ is gradient operator in $\omega$. We assume that $a_{i}(x)>0, i=1,2$, on $\bar{\omega}$, and $a_{i}, f_{i}$ and $g_{i}, i=1,2$, are sufficiently smooth functions.

In solving such nonlinear problems by the finite difference method, the corresponding discrete problem is usually formulated as a system of nonlinear algebraic equations. One then requires a reliable and efficient computational algorithm for computing the solution. A fruitful method for the treatment of these nonlinear systems is the method of upper and lower solutions and its associated monotone iterations [3].

Iterative domain decomposition algorithms based on Schwarz-type alternating procedures have received much attention for their potential as efficient 
algorithms for parallel computing. For solving nonlinear elliptic equations, Boglaev [1, 2] proposed the discrete iterative algorithms which combine the monotone approach and an iterative domain decomposition method based on the Schwarz alternating procedure. The computational domain is partitioned into many nonoverlapping subdomains with interface $\gamma$. Small interfacial subdomains are introduced near the interface $\gamma$, and approximate boundary values computed on $\gamma$ are used for solving problems on nonoverlapping subdomains. Thus, this approach may be considered as a variant of a block Gauss-Seidel iteration (or in the parallel context as a multi-coloured algorithm) for the subdomains with a Dirichlet-Dirichlet coupling through the interface variables. This article extends the monotone domain decomposition algorithms of Boglaev $[1,2]$ to the system of nonlinear elliptic equations (1).

Section 2 introduces a nonlinear finite difference scheme for the numerical solution of (1). Section 3 presents a monotone domain decomposition algorithm. We show that monotonic convergence is maintained under the proposed domain decomposition into nonoverlapping subdomains and associated algorithm. Section 4 applies the monotone domain decomposition to a gas-liquid interaction model.

\section{A nonlinear difference scheme}

On $\bar{\omega}^{x_{\alpha}}, \alpha=1, \ldots, k$, we set up nonuniform rectangular meshes

$$
\bar{\omega}^{h x_{\alpha}}=\left\{x_{\alpha}^{\left(i_{\alpha}\right)}, \quad 0 \leq i_{\alpha} \leq N_{\alpha} ; \quad x_{\alpha}^{(0)}=0, \quad x_{\alpha}^{\left(N_{\alpha}\right)}=r_{\alpha}\right\}, \quad \alpha=1, \ldots, k .
$$

Thus, we represent the mesh $\bar{\omega}^{h}$ in the form

$$
\bar{\omega}^{h}=\prod_{\alpha=1}^{k} \bar{\omega}^{h x_{\alpha}}, \quad \bar{\omega}^{h}=\omega^{h} \cup \partial \omega^{h},
$$

where $\omega^{h}$ and $\partial \omega^{h}$ are sets of interior and boundary mesh points, respectively. 
For solving (1), consider the nonlinear difference scheme in the canonical form [5]

$$
\begin{aligned}
& \mathcal{L}_{i}^{h} U_{i}(p)+f_{i}\left(p, U_{1}, U_{2}\right)=0, \quad p \in \omega^{h}, \quad U_{i}=g_{i} \text { on } \partial \omega^{h}, \quad i=1,2, \\
& \mathcal{L}_{i}^{h} U_{i}(p) \equiv d_{i}(p) U_{i}(p)-\sum_{p^{\prime} \in \sigma^{\prime}(p)} e_{i}\left(p, p^{\prime}\right) U_{i}\left(p^{\prime}\right),
\end{aligned}
$$

where $\sigma^{\prime}(p)=\sigma(p) \backslash\{p\}, \sigma(p)$ is a stencil of the scheme at an interior mesh point $p \in \omega^{h}$ and $\partial \omega^{h}$ is the boundary of $\bar{\omega}^{h}$. We make the following assumptions on the coefficients of the difference operators $\mathcal{L}_{i}^{h}, i=1,2$ :

$$
d_{i}(p)>0, \quad e_{i}\left(p, p^{\prime}\right) \geq 0, \quad d_{i}(p)-\sum_{p^{\prime} \in \sigma^{\prime}(p)} e_{i}\left(p, p^{\prime}\right) \geq 0, \quad p \in \omega^{h} .
$$

We now formulate the discrete maximum principle.

Lemma 1 Let the coefficients of the difference operators $\mathcal{L}_{i}^{h}, i=1,2$, in (2) satisfy (3). If mesh functions $W_{i}(p), i=1,2$, satisfy the conditions

$$
\left(\mathcal{L}_{i}^{h}+c_{i}\right) W_{i}(p) \geq 0(\leq 0), \quad p \in \omega^{h}, \quad W_{i}(p) \geq 0(\leq 0), \quad p \in \partial \omega^{h},
$$

where $c_{i}(p) \geq 0, i=1,2$, then $W_{i}(p) \geq 0(\leq 0), i=1,2$, in $\bar{\omega}^{h}$.

Samarskii [5] gave the proof of this lemma.

Remark 2 Difference schemes which satisfy the maximum principle from Lemma 1 are said to be monotone. The monotonicity condition guarantees that the systems of algebraic equations based on such methods are well-posed. 


\section{Monotone domain decomposition algorithm}

By hyperplane

$$
\left\{x_{1}=\rho_{m}, m=1, \ldots, M-1: \rho_{0}=0<\rho_{1}<\cdots<\rho_{M-1}<\rho_{M}=r_{1}\right\},
$$

we decompose the mesh $\bar{\omega}^{h}$ into $M$ nonoverlapping rectangular subdomains $\bar{\omega}_{m}^{h}$, $m=1, \ldots, M$ :

$$
\begin{aligned}
& \bar{\omega}^{h}=\bigcup_{m=1}^{M} \bar{\omega}_{m}^{h}, \quad \partial \omega_{m}^{h}=\gamma_{m}^{0} \cup \gamma_{m-1} \cup \gamma_{m}, \quad \gamma_{m}^{0}=\partial \omega^{h} \cap \bar{\omega}_{m}^{h}, \\
& \gamma_{m}=\left\{\rho_{m}\right\} \times \bar{\omega}^{h y}, \quad \bar{\omega}^{h y} \equiv \prod_{\alpha=2}^{k} \bar{\omega}^{h x_{\alpha}}, \quad \bar{\omega}_{m}^{h} \cap \bar{\omega}_{m+1}^{h}=\gamma_{m},
\end{aligned}
$$

where the boundary $\partial \omega_{m}^{h}$ of $\bar{\omega}_{m}^{h}$ consists of the boundaries $\gamma_{m-1}$ and $\gamma_{m}$ which belong to the hyperplane $\rho_{m-1}$ and $\rho_{m}$, respectively, and $\gamma_{m}^{0}$ which belongs to the boundary $\partial \omega^{h}$. On $\bar{\omega}^{h}$ we introduce $(M-1)$ interfacial subdomains $\bar{\vartheta}_{m}^{h}$, $m=1, \ldots, M-1$, with boundaries $\partial \vartheta_{m}^{h}$ in the form

$$
\begin{aligned}
& \partial \vartheta_{m}^{h}=\gamma_{m}^{c} \cup \gamma_{m}^{b} \cup \gamma_{m}^{e}, \quad \gamma_{m}^{c}=\partial \omega^{h} \cap \bar{\vartheta}_{m}^{h}, \\
& \gamma_{m}^{b}=\left\{\rho_{m}^{b}\right\} \times \bar{\omega}^{h y}, \quad \gamma_{m}^{e}=\left\{\rho_{m}^{e}\right\} \times \bar{\omega}^{h y}, \quad \rho_{m}^{b}<\rho_{m}<\rho_{m}^{e},
\end{aligned}
$$

where $\bar{\vartheta}_{m}^{h}$ overlaps $\bar{\omega}_{m}^{h} \cup \bar{\omega}_{m+1}^{h}$. Figure 1 illustrates the domain decomposition in the two dimensional case.

We assume that sizes of all the subdomains $\bar{\omega}_{m}^{h}$ and $\bar{\vartheta}_{m}^{h}$ allow us to solve Dirichlet boundary value problems based on the difference equations from (2).

\subsection{Statement of domain decomposition algorithm}

Consider the following domain decomposition approach for solving (2). On each iterative step, we first solve problems on the nonoverlapping subdo- 


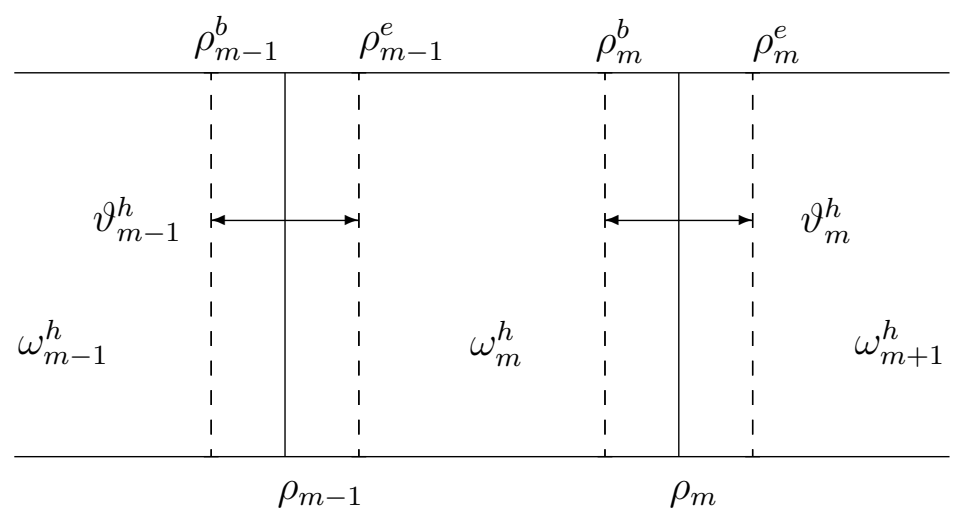

FiguRE 1: Fragment of the domain decomposition.

mains $\bar{\omega}_{m}^{h}, m=1, \ldots, M$, with Dirichlet boundary conditions passed from the previous iterate. Then Dirichlet data are passed from these subdomains to the interfacial subdomains $\bar{\vartheta}_{m}^{h}, m=1, \ldots, M-1$, and problems on the interfacial subdomains are computed. Finally, we piece together the solutions on the subdomains.

1. Initialization: On the whole mesh $\bar{\omega}^{h}$, choose initial functions $V_{i}^{(0)}(p)$, $p \in \bar{\omega}^{h}, i=1,2$.

2. On subdomains $\bar{\omega}_{m}^{h}, m=1, \ldots, M$, compute mesh functions $V_{i, m}^{(n)}(p)$, $m=1, \ldots, M, i=1,2$, (here the index $n$ stands for a number of iterative steps) satisfying the difference schemes

$$
\begin{aligned}
& \left(\mathcal{L}_{i}^{h}+\bar{c}_{i}\right) Z_{i, m}^{(n)}(p)=-\mathcal{R}_{i}^{h}\left(p, V_{1}^{(n-1)}, V_{2}^{(n-1)}\right), \quad p \in \omega_{m}^{h}, \\
& Z_{i, m}^{(1)}(p)= \begin{cases}g_{i}(p)-V_{i}^{(0)}(p), & p \in \gamma_{m}^{0}, \\
0, & p \in \gamma_{m-1} \cup \gamma_{m},\end{cases} \\
& Z_{i, m}^{(n)}(p)=0, \quad p \in \partial \omega_{m}^{h}, \quad n \geq 2,
\end{aligned}
$$




$$
\mathcal{R}_{i}^{h}\left(p, V_{1}^{(n-1)}, V_{2}^{(n-1)}\right) \equiv \mathcal{L}_{i}^{h} V_{i}^{(n-1)}+f_{i}\left(p, V_{1}^{(n-1)}, V_{2}^{(n-1)}\right),
$$

where mesh functions $\bar{c}_{i}(p), i=1,2$, is defined in (9).

3. On the interfacial subdomains $\bar{\vartheta}_{m}^{h}, m=1, \ldots, M-1$, compute the difference problems

$$
\left(\mathcal{L}_{i}^{h}+\bar{c}_{i}\right) Y_{i, m}^{(n)}(p)=-\mathcal{R}_{i}^{h}\left(p, V_{1}^{(n-1)}, V_{2}^{(n-1)}\right), \quad p \in \vartheta_{m}^{h},
$$

with the boundary conditions $Y_{i, m}^{(n)}\left(\gamma_{m}^{c}\right)=0, Y_{i, m}^{(n)}\left(\gamma_{m}^{b}\right)=Z_{i, m}^{(n)}\left(\gamma_{m}^{b}\right)$, $Y_{i, m}^{(n)}\left(\gamma_{m}^{e}\right)=Z_{i, m+1}^{(n)}\left(\gamma_{m}^{e}\right)$

4. Compute the mesh functions $V_{i}^{(n)}(p), p \in \bar{\omega}^{h}, i=1,2$, by piecing together the solutions on the subdomains

$$
V_{i}^{(n)}(p)= \begin{cases}V_{i, m}^{(n)}(p), & p \in \bar{\omega}_{m}^{h} \backslash\left(\bar{\vartheta}_{m-1}^{h} \cup \bar{\vartheta}_{m}^{h}\right), \quad m=1, \ldots, M, \\ W_{i, m}^{(n)}(p), & p \in \bar{\vartheta}_{m}^{h}, \quad m=1, \ldots, M-1,\end{cases}
$$

where $V_{i, m}^{(n)}=V_{i}^{(n-1)}+Z_{i, m}^{(n)}, W_{i, m}^{(n)}=V_{i}^{(n-1)}+Y_{i, m}^{(n)}$, and $\bar{\vartheta}_{0}^{h}=\emptyset$, $\bar{\vartheta}_{M}^{h}=\emptyset$.

5. Stopping criterion is

$$
\max _{p \in \bar{\omega}^{h}}\left|V_{i}^{(n)}(p)-V_{i}^{(n-1)}(p)\right| \leq \delta, \quad i=1,2,
$$

where $\delta$ is a prescribed accuracy. If the stopping criterion is reached, then stop, otherwise go to Step 2.

Algorithm (4)-(6) can be carried out by parallel processing, since on each iterative step $n$ the $2 M$ problems (4) for $Z_{i, m}^{(n)}(p), m=1, \ldots, M, i=1,2$, and the $2(M-1)$ problems $(5)$ for $Y_{i, m}^{(n)}(p), m=1, \ldots, M-1, i=1,2$, can be implemented concurrently. 


\subsection{Monotone convergence of algorithm (4)-(6)}

We assume that the functions $f_{i}, i=1,2$, are quasi-monotone nonincreasing

$$
-\partial f_{1} / \partial u_{2} \leq 0, \quad-\partial f_{2} / \partial u_{1} \leq 0 .
$$

Remark 3 Systems of elliptic equations with quasi-monotone nonincreasing reaction functions appear in a number of reaction-diffusion models including gas-liquid interaction models, the Belousov-Zhabotinskii reaction-diffusion model and the Volterra-Lotka competition model in ecology [4].

Vector mesh functions $\widetilde{\mathbf{U}}(p)=\left(\widetilde{U}_{1}(p), \widetilde{U}_{2}(p)\right)$ and $\widehat{\mathbf{U}}(p)=\left(\widehat{U}_{1}(p), \widehat{U}_{2}(p)\right)$ are called ordered upper and lower solutions of (2) if they satisfy the relation $\widetilde{\mathbf{U}}(p) \geq \widehat{\mathbf{U}}(p), p \in \bar{\omega}^{h}$, and if

$$
\begin{aligned}
& \mathcal{L}_{1}^{h} \widehat{U}_{1}(p)+f_{1}\left(p, \widehat{U}_{1}, \widetilde{U}_{2}\right) \leq 0 \leq \mathcal{L}_{1}^{h} \widetilde{U}_{1}(p)+f_{1}\left(p, \widetilde{U}_{1}, \widehat{U}_{2}\right), \quad p \in \omega^{h}, \\
& \mathcal{L}_{2}^{h} \widehat{U}_{2}(p)+f_{2}\left(p, \widetilde{U}_{1}, \widehat{U}_{2}\right) \leq 0 \leq \mathcal{L}_{2}^{h} \widetilde{U}_{2}(p)+f_{2}\left(p, \widehat{U}_{1}, \widetilde{U}_{2}\right), \quad p \in \omega^{h}, \\
& \widehat{U}_{i} \leq g_{i} \leq \widetilde{U}_{i} \text { on } \partial \omega^{h}, \quad i=1,2 .
\end{aligned}
$$

The ordering relation $\widetilde{\mathbf{U}} \geq \widehat{\mathbf{U}}$ is meant in the componentwise sense, that is, $\widetilde{U}_{i}(p) \geq \widehat{U}_{i}(p), i=1,2$.

We assume that $f_{i}, i=1,2$, satisfy the constraints

$$
\max \left\{\partial f_{i}\left(p, U_{1}, U_{2}\right) / \partial u_{i} ; \widehat{\mathbf{U}}(p) \leq \mathbf{U}(p) \leq \widetilde{\mathbf{U}}(p)\right\} \leq \bar{c}_{i}(p),
$$

where $\mathbf{U}=\left(U_{1}, U_{2}\right)$ and $\bar{c}_{i}(p) \geq 0, i=1,2$, are bounded functions in $\bar{\omega}^{h}$.

We get the following convergence property of algorithm (4)-(6). 
Theorem 4 Assume that the coefficients of the difference operators $\mathcal{L}_{i}^{h}, i=$ 1,2 , in (2) satisfy (3), the functions $f_{i}, i=1,2$, satisfy (7) and (9). Let $\widetilde{\mathbf{V}}$ and $\widehat{\mathbf{V}}$ be ordered upper and lower solutions (8) of (2).

If $\left(\bar{V}_{1}^{(0)}, \underline{V}_{2}^{(0)}\right)=\left(\widetilde{V}_{1}, \widehat{V}_{2}\right)$, then the sequence $\left\{\bar{V}_{1}^{(n)}, \underline{V}_{2}^{(n)}\right\}$ generated by (4)(6) converges monotonically to a solution $\left(\bar{V}_{1}, \underline{V}_{2}\right)$ of (2):

$$
\bar{V}_{1} \leq \bar{V}_{1}^{(n+1)} \leq \bar{V}_{1}^{(n)}, \quad \underline{V}_{2}^{(n)} \leq \underline{V}_{2}^{(n+1)} \leq \underline{V}_{2}, \quad \text { in } \bar{\omega}^{h} .
$$

If $\left(\underline{V}_{1}^{(0)}, \bar{V}_{2}^{(0)}\right)=\left(\widehat{V}_{1}, \widetilde{V}_{2}\right)$, then the sequence $\left\{\underline{V}_{1}^{(n)}, \bar{V}_{2}^{(n)}\right\}$ generated by (4)(6) converges monotonically to a solution $\left(\underline{V}_{1}, \bar{V}_{2}\right)$ of (2):

$$
\underline{V}_{1}^{(n)} \leq \underline{V}_{1}^{(n+1)} \leq \underline{V}_{1}, \quad \bar{V}_{2} \leq \bar{V}_{2}^{(n+1)} \leq \bar{V}_{2}^{(n)}, \quad \text { in } \bar{\omega}^{h} .
$$

Moreover, the corresponding iterations $\left(\bar{V}_{1}^{(n)}, \bar{V}_{2}^{(n)}\right)$ and $\left(\underline{V}_{1}^{(n)}, \underline{V}_{2}^{(n)}\right)$ are ordered upper and lower solutions.

Proof: Suppose that $\left(\bar{V}_{1}^{(0)}, \underline{V}_{2}^{(0)}\right)=\left(\widetilde{V}_{1}, \widehat{V}_{2}\right)$. By the maximum principle in Lemma 1, from (4) and (8), we conclude that

$$
Z_{1, m}^{(1)}(p) \leq 0, \quad Z_{2, m}^{(1)}(p) \geq 0, \quad p \in \bar{\omega}_{m}^{h} .
$$

Using the mean value theorem, from (4) we obtain for $i=1,2$,

$$
\mathcal{R}_{i}^{h}\left(p, V_{1, m}^{(1)}, V_{2, m}^{(1)}\right)=-\left(\bar{c}_{i}-\partial f_{i}^{(1)} / \partial u_{i}\right) Z_{i, m}^{(1)}+\left(\partial f_{i}^{(1)} / \partial u_{i^{\prime}}\right) Z_{i^{\prime}, m}^{(1)}, \quad p \in \omega_{m}^{h},
$$

where $i^{\prime} \neq i$, the variable $p$ is suppressed and the partial derivatives are calculated at intermediate points $\left(\eta_{i, m}, \mu_{i, m}\right)$ such that $V_{1, m}^{(1)} \leq \eta_{i, m} \leq \bar{V}_{1}^{(0)}$, $\underline{V}_{2}^{(0)} \leq \mu_{i} \leq V_{2, m}^{(1)}$. From here, (7), (9) and (10), we conclude

$$
\mathcal{R}_{1}^{h}\left(p, V_{1, m}^{(1)}, V_{2, m}^{(1)}\right) \geq 0, \quad \mathcal{R}_{2}^{h}\left(p, V_{1, m}^{(1)}, V_{2, m}^{(1)}\right) \leq 0, \quad p \in \omega_{m}^{h},
$$




$$
V_{i, m}^{(1)}(p)=g_{i}(p), \quad p \in \gamma_{m}^{0}, \quad V_{i, m}^{(1)}(p)=V_{i}^{(0)}(p), \quad p \in \gamma_{m-1} \cup \gamma_{m},
$$

where $V_{1}^{(0)}=\bar{V}_{1}^{(0)}$ and $V_{2}^{(0)}=\underline{V}_{2}^{(0)}$. Taking into account (10), by the maximum principle in Lemma 1, from (5) and (8), it follows that

$$
Y_{1, m}^{(1)}(p) \leq 0, \quad Y_{2, m}^{(1)}(p) \geq 0, \quad p \in \bar{\vartheta}_{m}^{h} .
$$

Similar to (12), we can prove

$$
\mathcal{R}_{1}^{h}\left(p, W_{1, m}^{(1)}, W_{2, m}^{(1)}\right) \geq 0, \quad \mathcal{R}_{2}^{h}\left(p, W_{1, m}^{(1)}, W_{2, m}^{(1)}\right) \leq 0, \quad p \in \vartheta_{m}^{h},
$$

where $W_{i, m}^{(1)}\left(\gamma_{m}^{c}\right)=g_{i}\left(\gamma_{m}^{c}\right), W_{i, m}^{(1)}\left(\gamma_{m}^{b}\right)=V_{i, m}^{(1)}\left(\gamma_{m}^{b}\right), W_{i, m}^{(1)}\left(\gamma_{m}^{e}\right)=V_{i, m+1}^{(1)}\left(\gamma_{m}^{e}\right)$. From here, (12) and the definition of $V_{i}^{(1)}, i=1,2$, we conclude that

$$
\mathcal{R}_{1}^{h}\left(p, V_{1}^{(1)}, V_{2}^{(1)}\right) \geq 0, \quad \mathcal{R}_{2}^{h}\left(p, V_{1}^{(1)}, V_{2}^{(1)}\right) \leq 0, \quad p \in \omega^{h} \backslash \gamma,
$$

where $\gamma=\bigcup_{m=1}^{M-1} \gamma_{m}^{b, e}$. From the boundary conditions for $V_{i, m}^{(1)}$ and $W_{i, m}^{(1)}$, $i=1,2$, it follows that $V_{i}^{(1)}, i=1,2$, satisfy the boundary conditions in (2). We now prove that the above inequalities hold true on the interfacial boundaries $\gamma_{m}^{b}$ and $\gamma_{m}^{e}, m=1, \ldots, M-1$. We check these inequalities in the case of the left boundary $\gamma_{m}^{b}$, since the second case is checked in a similar way. From (4), (5), (10) and (13), we conclude that the mesh functions $R_{i, m}^{(1)}=V_{i, m}^{(1)}-W_{i, m}^{(1)}, i=1,2$, satisfy the difference problems

$$
\begin{aligned}
& \left(\mathcal{L}_{i}^{h}+\bar{c}_{i}\right) R_{i, m}^{(1)}(p)=0, \quad p \in \vartheta_{m}^{h b}=\omega_{m}^{h} \cap \vartheta_{m}^{h}, \\
& R_{i, m}^{(1)}(p)=0, \quad p \in \partial \vartheta_{m}^{h b} \backslash \gamma_{m}, \quad R_{1, m}^{(1)}(p) \geq 0, \quad R_{2, m}^{(n)}(p) \leq 0, \quad p \in \gamma_{m} .
\end{aligned}
$$

In view of the maximum principle in Lemma 1 ,

$$
V_{1, m}^{(1)}(p)-W_{1, m}^{(1)}(p) \geq 0, \quad V_{2, m}^{(1)}(p)-W_{2, m}^{(1)}(p) \leq 0, \quad p \in \bar{\vartheta}_{m}^{h b}, \quad i=1,2 .
$$

From here, (3), (6), (12), and taking into account that $W_{i, m}^{(n)}(p)=V_{i, m}^{(n)}(p)$, $p \in \gamma_{m}^{b}, i=1,2$, it follows that

$$
\mathcal{R}_{1}^{h}\left(p, V_{1}^{(1)}, V_{2}^{(1)}\right) \geq \mathcal{R}_{1}^{h}\left(p, V_{1, m}^{(1)}, V_{2, m}^{(1)}\right) \geq 0, \quad p \in \gamma_{m}^{b},
$$




$$
\mathcal{R}_{2}^{h}\left(p, V_{1}^{(1)}, V_{2}^{(1)}\right) \leq \mathcal{R}_{2}^{h}\left(p, V_{1, m}^{(1)}, V_{2, m}^{(1)}\right) \leq 0, \quad p \in \gamma_{m}^{b}
$$

This leads to the fact that $\bar{V}_{1}^{(1)}$ and $\underline{V}_{2}^{(1)}$ are upper and lower solutions of problem (2), respectively. From (10) and (13), we have $\bar{V}_{1}^{(1)} \leq \bar{V}_{1}^{(0)}, \underline{V}_{2}^{(1)} \geq \underline{V}_{2}^{(0)}$. By induction on $n$, we can prove that $\left\{\bar{V}_{1}^{(n)}\right\}$ and $\left\{\underline{V}_{2}^{(n)}\right\}$ are monotonically decreasing and increasing sequences of upper and lower solutions to problem (2), respectively.

Similarly, for $\left(\underline{V}_{1}^{(0)}, \bar{V}_{2}^{(0)}\right)=\left(\widehat{V}_{1}, \widetilde{V}_{2}\right)$, we can prove that $\left\{\underline{V}_{1}^{(n)}\right\}$ and $\left\{\bar{V}_{2}^{(n)}\right\}$ are monotonically increasing and decreasing sequences of lower and upper solutions to problem (2), respectively.

We now prove that $\left(\bar{V}_{i}^{(n)}, \underline{V}_{i}^{(n)}\right), i=1,2$, are ordered upper and lower solutions

$$
\underline{V}_{i}^{(n)}(p) \leq \bar{V}_{i}^{(n)}(p), \quad p \in \bar{\omega}^{h}, \quad i=1,2 .
$$

Introduce the notation $Q_{i}^{(n)}=\bar{V}_{i}^{(n)}-\underline{V}_{i}^{(n)}, i=1,2$. Similar to (11), using the mean-value theorem, from (4)-(6) with $i=1$ and $n=1$, (7), (9) and taking into account that $\widetilde{\mathbf{V}}$ and $\widehat{\mathbf{V}}$ are ordered upper and lower solutions to (2), we obtain

$$
\left(\mathcal{L}_{1}^{h}+\bar{c}_{1}\right) Q_{1}^{(1)}=\left(\bar{c}_{i}-\partial f_{1}^{(1)} / \partial u_{1}\right) Q_{1}^{(0)}+\left(\partial f_{1}^{(1)} / \partial u_{2}\right) Q_{2}^{(0)} \geq 0, \quad p \in \omega^{h} \backslash \gamma
$$

$Q_{1}^{(1)}(p)=0, \quad p \in \partial \omega^{h}$.

We prove that

$$
\left(\mathcal{L}_{1}^{h}+\bar{c}_{1}\right) Q_{1}^{(1)} \geq 0, \quad p \in \gamma
$$

We check this inequality on $\gamma_{m}^{b}$. Similar to $(17)$, in the notation $Q_{i, m}^{(n)}=$ $\bar{V}_{i, m}^{(n)}-\underline{V}_{i, m}^{(n)}$, we can get

$$
\left(\mathcal{L}_{1}^{h}+\bar{c}_{1}\right) Q_{1, m}^{(1)}=\left(\bar{c}_{i}-\partial f_{1}^{(1)} / \partial u_{1}\right) Q_{1}^{(0)}+\left(\partial f_{1}^{(1)} / \partial u_{2}\right) Q_{2}^{(0)} \geq 0, \quad p \in \omega_{m}^{h} .
$$

From here, (3), (6), (12), (15), the inverse inequalities in (15) for the case $\left(\underline{V}_{1}^{(0)}, \bar{V}_{2}^{(0)}\right)=\left(\widehat{V}_{1}, \widetilde{V}_{2}\right)$, and taking into account that $W_{i, m}^{(n)}(p)=V_{i, m}^{(n)}(p), p \in$ 
$\gamma_{m}^{b}, i=1,2$, it follows that

$$
\left(\mathcal{L}_{1}^{h}+\bar{c}_{1}\right) Q_{1}^{(1)} \geq\left(\mathcal{L}_{1}^{h}+\bar{c}_{1}\right) Q_{1, m}^{(1)} \geq 0, \quad p \in \gamma_{m}^{b} .
$$

From here and (17), by the maximum principle in Lemma 1, we conclude (16) for $i=1, n=1$. Now, by induction on $n$, we can prove (16) for $i=1$. In the same way, we can prove (16) for $i=2$ and $n \geq 1$.

From (16), we conclude that the monotonically decreasing and increasing sequences $\left\{\bar{V}_{i}^{(n)}\right\},\left\{\underline{V}_{i}^{(n)}\right\}, i=1,2$, are bounded from below and above, respectively, by any lower and upper solutions, and, hence, are convergent

$$
\lim _{n \rightarrow \infty} \bar{V}_{i}^{(n)}(p)=\bar{V}_{i}(p), \quad \lim _{n \rightarrow \infty} \underline{V}_{i}^{(n)}(p)=\underline{V}_{i}(p), \quad p \in \bar{\omega}^{h} .
$$

From here, linearity of $\mathcal{L}_{i}^{h}, i=1,2$, and continuity of $f_{i}, i=1,2$, we conclude that $\left(\bar{V}_{1}, \underline{V}_{2}\right)$ and $\left(\underline{V}_{1}, \bar{V}_{2}\right)$ are solutions to (2). This completes the proof of the theorem.

\section{A gas-liquid interaction model}

Consider the gas-liquid interaction model where a dissolved gas $A$ and a dissolved reactant $B$ interact in a bounded diffusion medium $\omega$. The chemical reaction scheme is $A+\kappa_{1} B \rightarrow \kappa_{2} P$ and is called a second order reaction, where $\kappa_{1}$ and $\kappa_{2}$ are the rate constants and $P$ is the product. Denoting by $u_{1}(x)$ and $u_{2}(x)$ the concentrations of the dissolved gas $A$ and the reactant $B$, respectively, the above reaction scheme is governed by (1) with $L_{i} u_{i}=D_{i} \Delta u_{i}, f_{i}=\sigma_{i} u_{1} u_{2}, i=1,2$, where $D_{i}>0, i=1,2$, are the diffusion coefficients, and $\sigma_{1}$ is the rate constant, $\sigma_{2}=\kappa_{1} \sigma_{1}$. In a more general reaction scheme called the $(l, s)$ th order reaction, the resulting equations are given by (1) with

$$
f_{i}\left(x, u_{1}, u_{2}\right)=\sigma_{i} u_{1}^{l} u_{2}^{s}-q_{i}(x), \quad i=1,2,
$$


where $l \geq 1, s \geq 1$ are constants, $\sigma_{i}>0, i=1,2$, are the rate constants and $q_{i}(x) \geq 0, i=1,2$, are possible internal sources [4]. The system (1) reduces to

$$
-D_{i} \Delta u_{i}+\sigma_{i} u_{1}^{l} u_{2}^{s}-q_{i}(x)=0, \quad x \in \omega, \quad u_{i}=g_{i} \geq 0 \text { on } \partial \omega, \quad i=1,2 .
$$

The functions $f_{1}$ and $f_{2}$ are quasi-monotone nonincreasing in $\left(\mathbb{R}^{k}\right)^{+} \times\left(\mathbb{R}^{k}\right)^{+}$.

The nonlinear difference scheme (2) reduces to

$$
\mathcal{L}_{i}^{h} U_{i}(p)+f_{i}\left(p, U_{1}, U_{2}\right)=0, \quad p \in \omega^{h}, \quad U_{i}=g_{i} \text { on } \partial \omega^{h}, \quad i=1,2,
$$

where $f_{i}, i=1,2$, are defined by (18) and $\mathcal{L}_{i}^{h}, i=1,2$, are approximations of $D_{i} \Delta, i=1,2$, on a mesh $\bar{\omega}^{h}$. We assume that the coefficients of the difference operators $\mathcal{L}_{i}^{h}, i=1,2$, satisfy (3).

Consider the mesh functions $\left(\widetilde{U}_{1}, \widehat{U}_{2}\right)=\left(W_{1}, 0\right)$ and $\left(\widehat{U}_{1}, \widetilde{U}_{2}\right)=\left(0, W_{2}\right)$, where $W_{1}$ and $W_{2}$ solve the problems

$$
\mathcal{L}_{i}^{h} W_{i}(p)=q_{i}(p), \quad p \in \omega^{h}, \quad W_{i}(p)=g_{i}(p), \quad p \in \partial \omega^{h}, \quad i=1,2 .
$$

From here, (20) and taking into account that $f_{i}=0, i=1,2$, on $\left(W_{1}, 0\right)$ and $\left(0, W_{2}\right)$, we conclude that $\left(\widetilde{U}_{1}, \widehat{U}_{2}\right)=\left(W_{1}, 0\right)$ and $\left(\widehat{U}_{1}, \widetilde{U}_{2}\right)=\left(0, W_{2}\right)$ satisfy conditions (8). By Lemma 1, from (21) we conclude that $W_{i} \geq 0, i=1,2$. From the above construction, we conclude that

$$
\widetilde{\mathbf{U}}(p)=\left(W_{1}(p), W_{2}(p)\right), \quad \widehat{\mathbf{U}}(p)=(0,0), \quad p \in \bar{\omega}^{h},
$$

are ordered upper and lower solutions of (2).

From (19), we conclude that in $\{\widehat{\mathbf{U}}(p) \leq \mathbf{U}(p) \leq \widetilde{\mathbf{U}}(p)\}$

$\partial f_{1}\left(p, U_{1}, U_{2}\right) / \partial u_{1}=\sigma_{1} l U_{1}^{l-1}(p) U_{2}^{s}(p) \leq \sigma_{1} l W_{1}^{l-1}(p) W_{2}^{s}(p), \quad p \in \bar{\omega}^{h}$,

$\partial f_{2}\left(p, U_{1}, U_{2}\right) / \partial u_{2}=\sigma_{2} s U_{1}^{l}(p) U_{2}^{s-1}(p) \leq \sigma_{2} s W_{1}^{l}(p) W_{2}^{s-1}(p), \quad p \in \bar{\omega}^{h}$. 
From here, it follows that the assumptions in (9) hold true with

$$
\bar{c}_{1}(p)=\sigma_{1} l W_{1}^{l-1}(p) W_{2}^{s}(p), \quad \bar{c}_{2}(p)=\sigma_{2} s W_{1}^{l}(p) W_{2}^{s-1}(p) .
$$

Thus, the monotone domain decomposition algorithm (4)-(6) with the above constructed ordered upper and lower solutions and the coefficients $\bar{c}_{i}$, $i=1,2$, satisfies Theorem 4 for solving the gas-liquid interaction model (19).

\subsection{Numerical example}

Consider the gas-liquid system (19) in the case of the second order reaction with $l=s=1$ and zero internal sources. From (19), we write down the two-dimensional test problem in the non-dimensional variables

$$
\begin{aligned}
& -\left(\frac{\partial^{2} u_{1}}{\partial x_{1}^{2}}+\frac{\partial^{2} u_{1}}{\partial x_{2}^{2}}\right)+\sigma_{1} u_{1} u_{2}=0, \quad\left(x_{1}, x_{2}\right) \in \omega, \quad u_{1}=1 \text { on } \partial \omega \\
& -\mu^{2}\left(\frac{\partial^{2} u_{2}}{\partial x_{1}^{2}}+\frac{\partial^{2} u_{2}}{\partial x_{2}^{2}}\right)+\sigma_{2} u_{1} u_{2}=0, \quad\left(x_{1}, x_{2}\right) \in \omega, \quad u_{2}=1 \text { on } \partial \omega, \\
& \omega=\left\{0<x_{1}<1,0<x_{2}<1\right\}, \quad \mu^{2}=\frac{D_{2}}{D_{1}},
\end{aligned}
$$

where $u_{1}$ and $u_{2}$ are the non-dimensional concentrations of the dissolved gas $A$ and the reactant $B$, respectively. Since $\mu^{2} \ll 1$, the test problem is singularly perturbed and $u_{2}$ has boundary layers, (that is, regions with rapid change of the solution) near $\partial \omega$ of width $\mathcal{O}(\mu)$.

For the differential operators, we use the central difference approximations on the five point rectangular stencil, which satisfy (3). We employ a layer adapted mesh of a piecewise uniform type. The piecewise uniform mesh is formed by the following manner. We divide each of the intervals $\bar{\omega}^{x_{1}}=[0,1]$ and $\bar{\omega}^{x_{2}}=[0,1]$ into three parts each $[0, \tau],[\tau, 1-\tau]$ and $[1-\tau, 1]$. Assuming that $N_{1}=N_{2}=N$ and $N$ is divisible by 4 , in the parts $[0, \tau],[1-\tau, 1]$ we 
use the uniform mesh with $N / 4+1$ mesh points, and in the part $[\tau, 1-\tau]$ with $N / 2+1$ mesh points. This defines the piecewise uniform mesh in the $x_{1}$ - and $x_{2}$-directions. The transition points $\tau$ and $1-\tau$ are determined by

$$
\tau=\min \left\{4^{-1}, \mu \ln N\right\} .
$$

If the parameter $\mu$ is small enough, then the uniform mesh inside of the boundary layers with the step size $h_{\mu}=4 \tau N^{-1}$ is fine, and the uniform mesh outside of the boundary layers with the step size $h=2(1-2 \tau) N^{-1}$ is coarse. The central difference schemes on the piecewise uniform mesh converge $\mu$ uniformly to the solution of the test problem.

From (21)-(23), we conclude that

$$
\widetilde{\mathbf{U}}(p)=(1,1), \quad \widehat{\mathbf{U}}(p)=(0,0), \quad p \in \bar{\omega}^{h}, \quad \bar{c}_{i}=\sigma_{i}, \quad i=1,2 .
$$

We choose $\sigma_{1}=2, \sigma_{2}=1$ and $\delta=10^{-5}$ in the stopping criterion. We use balanced domain decompositions, where the mesh points are equally distributed among the main subdomains, and solve all linear systems in the monotone domain decomposition algorithm (4)-(6) by ICCG-method. The initial guess is

$$
\bar{V}_{1}^{(0)}(p)=1, \quad p \in \bar{\omega}^{h}, \quad \underline{V}_{2}^{(0)}(p)=0, \quad p \in \bar{\omega}^{h} .
$$

Our numerical results show that for all values of $\mu$ and $N$ and all domain decompositions, the sequence $\left\{\bar{V}_{1}^{(n)}, \underline{V}_{2}^{(n)}\right\}$ generated by (4)-(6) converges monotonically to the exact solution of the nonlinear difference scheme. This confirms the theoretical results in Theorem 4.

For $\mu \leq 10^{-2}$, the convergence iteration count for the monotone undecomposed algorithm with $M=1$ is uniform with respect to $\mu$ and $N$ and equals three. Our numerical results show, that if $\mu \leq 10^{-2}$, then for $N$ and $M$ fixed, the number of iterations $n_{0}$, required to reach the prescribed accuracy $\delta$, is independent of $\mu$. Table 1 , for various values of $N$ and $M$, gives 
TABLE 1: Number of iterations $n_{0}$ for algorithm (4)-(6).

\begin{tabular}{cccccc}
\hline$M \backslash N$ & 32 & 64 & 126 & 256 & 512 \\
\hline 4 & 3 & 3 & 3 & 3 & 3 \\
8 & 7 & 6 & 5 & 5 & 4 \\
16 & 21 & 18 & 15 & 13 & 11 \\
32 & n.a. & 53 & 44 & 36 & 28
\end{tabular}

the number of iterations $n_{0}$ for the monotone domain decomposition algorithm (4)-(6). From Table 1, we conclude that for $M$ fixed, the number of iterations is a monotone decreasing function with respect to the number of mesh points $N$. For $M \leq 8$, the numbers of iterations for the monotone domain decomposition algorithm (4)-(6) are almost the same as for the monotone undecomposed algorithm. The last numerical results are important in the context of parallel implementation of the monotone domain decomposition algorithm (4)-(6).

\section{References}

[1] I. Boglaev. On monotone iterative methods for a nonlinear singularly perturbed reaction-diffusion problem. J. Comput. Appl. Math., 162:445-466, 2004. doi:10.1016/j.cam.2003.08.035 C593

[2] I. Boglaev. Monotone iterates for solving nonlinear monotone difference schemes. Computing, 78:17-30, 2006. doi:10.1007/s00607-006-0168-0 C593

[3] C. V. Pao. Monotone iterative methods for finite difference systems of reaction-diffusion equations. Numer. Math. 46:571-586, 1985. doi:10.1007/BF01389659 C592 
[4] C. V. Pao. Nonlinear Parabolic and Elliptic Equations. Plenum Press, New York, 1992. C598, C603

[5] A. Samarskii. The Theory of Difference Schemes. Marcel Dekker Inc., New York-Basel, 2001. C594 


\section{Author address}

1. I. Boglaev, Institute of Fundamental Sciences, Massey University, Palmerston North, New ZEALAND mailto:i.boglaev@massey.ac.nz 